\title{
Análise bibliométrica da produção científica da indústria 4.0 e sua relação com a indústria calçadista
}

\author{
A bibliometric analysis of the scientific production of industry 4.0 and its relationship with the \\ footwear industry \\ Análisis bibliométrico de la producción cientifica de la industria 4.0 y su relación con la industria \\ del calzado
}

Frederico Romel Maia Tavares ORCID: https://orcid.org/0000-0001-5156-2167 Universidade Regional do cariri, Brasil E-mail: prof_fred@hotmail.com

Francisco Bruno Juvino Da Cruz ORCID: https://orcid.org/0000-0001-6622-7783 Universidade Regional do cariri, Brasil E-mail: cruzbruno596@gmail.com

Caio Vinicius De Araujo Ferreira Gomes ORCID: https://orcid.org/0000-0002-4952-2454 Universidade Regional do cariri, Brasil E-mail: caioviniciusa2@gmail.com

Caio Vinicius De Araujo Ferreira Gomes ORCID: https://orcid.org/0000-0002-4952-2454 Universidade Regional do cariri, Brasil E-mail: caioviniciusa2@gmail.com

José Francisco De Sousa Figueredo ORCID: https://orcid.org/0000-0002-1772-0878 Universidade Regional do cariri, Brasil E-mail: jfengenharia56@gmail.com

\begin{abstract}
Resumo
Este artigo buscou mostrar o crescimento da produção científica e mapear os principais estudos referentes à indústria 4.0 nos últimos cinco anos, evidenciando as suas principais características e tecnologias. O trabalho mostrou a relação do tema com uma das indústrias mais tradicionais e responsáveis pelo desenvolvimento regional em diversos países, a indústria de calçados. $\mathrm{O}$ trabalho também revelou a relação com a indústria 4.0 mostrando que existem ainda lacunas, e possibilidades de estudos promissores envolvendo os temas principalmente na área de design para indústria calçadista. A pesquisa foi realizada na base Web of Science a qual apontou os principais artigos publicados no período. Foi realizada uma revisão sistemática da literatura que juntamente com o auxílio da técnica bibliométrica, permitiu mostrar a ascendência dos temas estudados. É possível afirmar que o tema indústria 4.0 tem cada vez mostrado a sua relevância com o crescimento das pesquisas. $\mathrm{O}$ engajamento da indústria de calçados com a implementação de ferramentas da indústria 4.0 pode vir a permitir avanços na formulação de estratégias competitivas para o setor.
\end{abstract}

Palavras-chave: Indústria 4.0; Indústria calçadista; Bibliometria; Análise sistemática.

\begin{abstract}
This article sought to show the growth of scientific production and to map the main studies referring to industry 4.0 in the last five years, highlighting its main characteristics and technologies. The work showed the relationship of the theme with one of the most traditional industries responsible for regional development in several countries, the footwear industry. The work also revealed the relationship with industry 4.0, showing that there are still gaps, and possibilities for promising studies involving themes mainly in the area of desing for the footwear industry. The research was carried out on the Web of Science database, which pointed out the main articles published in the period. A systematic review of the literature was carried out to show the ancestry of the studied themes. It is possible to affirm that the theme of industry 4.0 has increasingly shown its relevance with the growth of research. The engagement of the footwear industry with the implementation of industry 4.0 tools may allow advances in the formulation of competitive strategies for the sector.
\end{abstract}

Keywords: Industry 4.0; Footwear industry; Bibliometrics; Systematic analysis. 


\begin{abstract}
Resumen
Este artículo buscó mostrar el crecimiento de la producción científica y mapear los principales estudios cinco años, destacando sus principales características y tecnologías. El trabajo mostró la relación del tema con una de las industrias más tradicionales responsable del desarrollo regional en varios países, la industria del calzado. El trabajo también reveló la relación con la industria 4.0, mostrando que aún existen brechas y posiblidades de estudios prometedores que involucren temas principalmente en el área del diseño para la industria del calzado. La investigación se llevó a cabo en la base de datos Web of Science, que señaló los principales artículos publicados en el período. Se realizó una revisión bibliográfica sistemática que, junto con la ayuda de la técnica bibliométrica, permitió evidenciar la ascendencia de los temas estudiados. Es posible afirmar que el tema de la industria 4.0 ha mostrado cada vez más su relevancia con el crecimiento de la investigación. El compromiso de la industria del calzado con la implementación de herramientas de la insdutria 4.0 puede permitir avances en la formulación de estrategias competitivas para el sector.
\end{abstract}

Palabras clave: Industria 4.0; Industria del calzado; Bibliometría; Análisis sistemático.

\title{
1. Introdução
}

As revoluções industriais ocorrem à medida que surgem novos avanços tecnológicos significativos e são adotados pelas fábricas, mudando assim toda a dinâmica produtiva. A primeira revolução industrial foi à passagem do processo de fabricação artesanal para o uso de máquinas a vapor. Em seguida, veio à segunda revolução, a qual foi marcada pela utilização da energia elétrica, combustíveis derivados do petróleo e a invenção do motor a explosão. Já a terceira revolução industrial foi definida, principalmente, pela adoção do computador.

Nesse contexto, muito ainda se discute sobre o que seria a quarta revolução industrial ou Indústria 4.0, quais as tecnologias que a compõem, bem como os benefícios que trará. Para Bär et al., (2018), ainda não existe um único conceito acerca da quarta revolução industrial, porém, a literatura ressalta o impacto na indústria e na concorrência internacional que as tecnologias que a integram acarretam. Assim, segundo Lasi et al., (2014), uma possível definição é que a indústria 4.0 é a adoção ao processo produtivo a combinação dos avanços tecnológicos baseados na integração da internet com dispositivos físicos atendendo a demanda dos clientes bem como a escassez dos recursos.

Diversos setores industriais já iniciaram a implementação dos conceitos e tecnologias referente à atual revolução industrial, entre elas a tradicional indústria de calçados a qual possui sua produção baseada em grande parte no trabalho manual, assim, exigindo uma grande quantidade de mão-de-obra. Essas novas tecnologias empregadas podem revolucionar o modo de produção com emprego de ferramentas como: análise de big data, CPS - Cyber Physical System, IoT - Internet of Things, impressão e scanner $3 d$, dentre outras. A indústria calçadista atua em um ambiente muito competitivo, e muitas dessas ferramentas podem promover o aumento dessa competitividade proporcionada pela redução de custos de produção, redução de mão-de-obra, redução no tempo de produção entre outros como ressaltam trabalhos recentes sobre o tema (Bai et al., 2020; Chang, 2020; Di Roma, 2017; Garzoni et al., 2020; Janson et al., 2019).

A análise bibliométrica é uma ferramenta robusta que possibilita o mapeamento e análise do desempenho acadêmico possibilitando entender a correlação entre os autores, disciplinas e estudos. Logo, esta ferramenta tem se tornado cada vez mais popular, visto que ela possibilita a obtenção de insights do meio acadêmico. (Gil et al., 2020; Kipper et al., 2020).

Este artigo tem por objetivo mapear os temas mais abordados nos últimos 5 (cinco) anos a respeito da indústria 4.0, mostrar a sua relação com a indústria de calçados e propor caminhos para pesquisas futuras correlacionando os dois temas.

\section{Metodologia}

O artigo caracterizasse como uma pesquisa quantitativa. Segundo Pereira et al. (2018), o método quantitativo trabalha com um grande fluxo de dados quantitativos ou numéricos que se utilizando de técnicas matemáticas para possibilitar análises detalhadas e previsões de acontecimentos. 
A revisão sistemática da literatura é uma ferramenta de pesquisa que tem por objetivo encontrar contribuições cientificas expandindo o banco de dados bibliográficos do pesquisador relacionado ao assunto, assim, evitando reinvenções de soluções já existentes. (Gil et al., 2020).

Neste presente artigo foi selecionado um método de revisão sistemática da literatura. Esse método ajudou a obter informações importantes sobre a Indústria 4.0 e a sua relação com a indústria de calçados. Para realizar a pesquisa, foi necessário utilizar a base de dados Web of Science referente aos últimos cinco anos.

Após os primeiros refinos foram escolhidos os 20 principais artigos referentes à indústria 4.0 tomando como critério de escolha o número de citações. Em seguida, foi realizado o mapeamento dos principais assuntos de interesse separados por clusters com o auxílio de software VOSViewer e uma descrição dos assuntos por periódicos.

Finalmente, fizemos um segundo refino na base de dados buscando identificar a relação entre à indústria 4.0 e a indústria de calçados. Após isso, foi realizado um mapeamento para identificar os principais assuntos de interesse, separados por cluster utilizando o software VOSViewer.

\section{Revisão Sistemática da Literatura - Indústria 4.0}

A indústria 4.0 tem se tornado objeto de estudos, intensificados nos últimos 5 anos, a partir da disseminação dos seus conceitos. Conforme Lasi et al., (2014), a evolução da industrialização passa por o que hoje se define como indústria 4.0 na visão de produção futura com sistemas de fabricação modulares e eficientes caracterizados por processos dos quais o controle da produção com amparo tecnológico se torna cada vez mais presente.

Inicialmente foi realizada uma busca na base de dados da Web of Science, usando apenas o tópico "Industry 4.0", revelando um total de 4.495 documentos publicados, considerando todo o período da base de dados até a data da busca. A produção técnica-cientifica com a temática indústria 4.0 iniciou-se em 2013 com apenas 01 artigo publicado, porém, foi apenas em 2017 que a produção ganhou destaque com um aumento de mais de 200\% em relação ao ano anterior.

A revisão sistemática da literatura foi preparada buscando identificar o grau de relevância do assunto presente nas pesquisas realizadas, as principais palavras-chaves e características, possibilitando assim apontar aspectos relevantes na área e potenciais linhas de pesquisa. O primeiro filtro empregado limitou a pesquisa para os documentos publicados nos últimos cinco anos, mostrando um crescimento significativo no número de citações, conforme mostra o Gráfico 1. 
Gráfico 1. Número de citações.

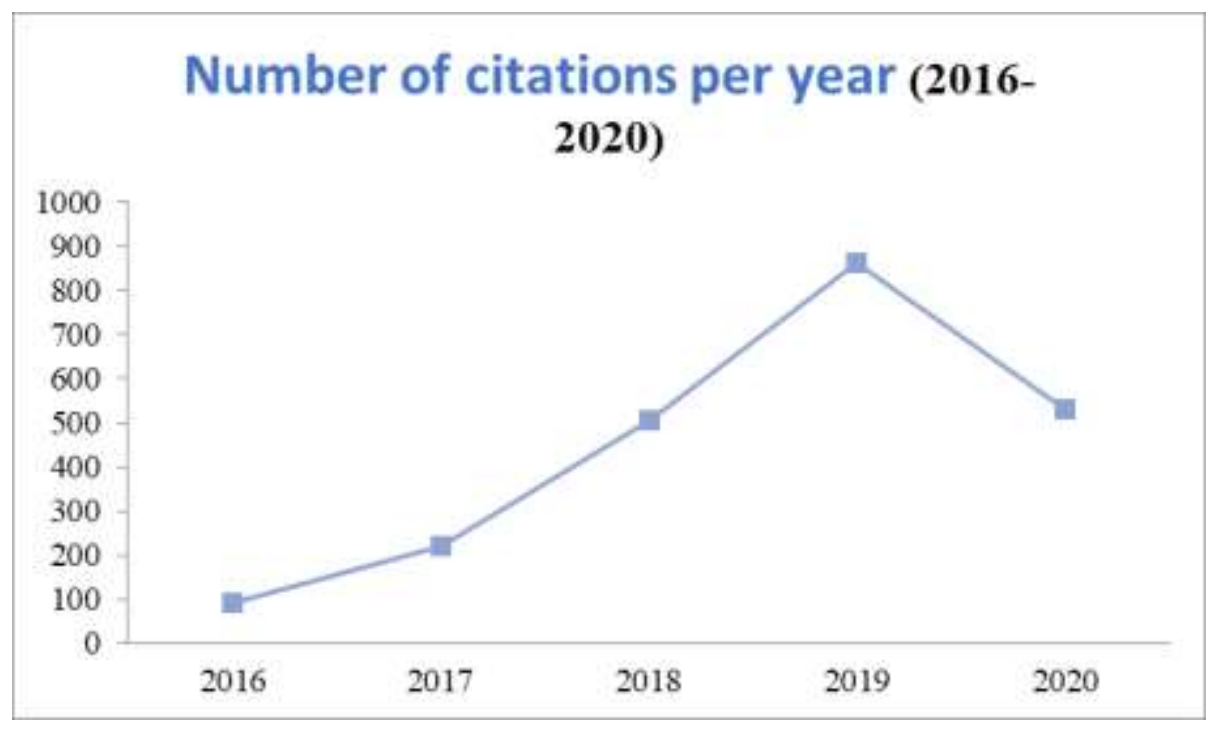

Fonte: Web of Science (2020).

Vale a pena salientar que quando foi feita a análise referente às áreas do conhecimento as informações gerais da amostra indicam uma dispersão, isso é indicativo da proporção que o estudo sobre indústria 4.0 já alcançou desde 2013. A Tabela 1 mostra a distribuição de citações por campo do conhecimento destacando-se: Engineering electrical electronic, Engineering industrial, Engineering manufacturing. Citações nos campos de: Engineering industrial, Engineering manufacturing. Além de citações nos campos operations research management, engeneering multidisciplinar e management, mostram a importância do projeto proposto.

Tabela 1. Distribuição de citações por campo do conhecimento.

\begin{tabular}{|c|c|c|}
\hline Campo: Categorias da Web of Science & Contagem do registro & $\%$ de 4,495 \\
\hline ENGINEERING ELECTRICAL ELECTRONIC & 909 & $20.222 \%$ \\
\hline ENGINEERING INDUSTRIAL & 706 & $15.706 \%$ \\
\hline ENGINEERING MANUFACTURING & 671 & $14.928 \%$ \\
\hline AUTOMATION CONTROL SYSTEMS & 531 & $11.813 \%$ \\
\hline COMPUTER SCIENCE THEORY METHODS & 470 & $10.456 \%$ \\
\hline COMPUTER SCIENCE INFORMATION SYSTEMS & 413 & $9.188 \%$ \\
\hline $\begin{array}{c}\text { COMPUTER SCIENCE INTERDISCIPLINARY } \\
\text { APPLICATIONS }\end{array}$ & 371 & $8.254 \%$ \\
\hline COMPUTER SCIENCE ARTIFICIAL INTELLIGENCE & 361 & $8.031 \%$ \\
\hline TELECOMMUNICATIONS & 323 & $7.186 \%$ \\
\hline OPERATIONS RESEARCH MANAGEMENT SCIENCE & 270 & $6.007 \%$ \\
\hline ENGINEERING MULTIDISCIPLINARY & 245 & $5.451 \%$ \\
\hline MANAGEMENT & 231 & $5.139 \%$ \\
\hline ENGINEERING MECHANICAL & 171 & $3.804 \%$ \\
\hline
\end{tabular}


Fonte: Web of Science (2020).

Após sete anos da introdução do termo "indústria 4.0" algumas obras destacaram-se por ter mais influência em decorrência da quantidade de citações que receberam de outros documentos. Desse modo, o artigo mais citado foi Industry 4.0 (Lasi et al., 2014) publicado na Busines \& Information Systems com o total de 18.714 citações.

Posteriormente um segundo filtro foi adicionado para permitir foco nos artigos relacionados com a pesquisa proposta. Selecionadas como categorias da Web of Science: Engineering industrial, regional urban planning, Engineering manufacturing, Operations research, Management science, Management or business, Economics e industrial relations labor. O resultado apontou um total de 76 artigos, nos quais os 20 mais citados estão dispostos no quadro abaixo:

Quadro 1. Artigos em ordem de número de citações.

\begin{tabular}{|c|c|c|c|}
\hline $\mathrm{N}^{\mathrm{o}}$ & Artigo & Autor/Ano & Revista \\
\hline 01 & $\begin{array}{l}\text { Business model innovation in small- and } \\
\text { medium-sized enterprises Strategies for } \\
\text { industry } 4.0 \text { providers and users }\end{array}$ & $\begin{array}{l}\text { Mueller, Julian Marius } \\
\text { (2019). }\end{array}$ & $\begin{array}{l}\text { Journal of manufacturing } \\
\text { technology management }\end{array}$ \\
\hline 02 & $\begin{array}{c}\text { Identifying Industry 4.0 IoT enablers by } \\
\text { integrated PCA-ISM-DEMATEL approach }\end{array}$ & $\begin{array}{l}\text { Rajput, Shubhangini; Singh, Surya } \\
\text { Prakash (2019). }\end{array}$ & Management decision \\
\hline 03 & $\begin{array}{c}\text { Exploring Industry } 4.0 \text { technologies to enable } \\
\text { circular economy practices in a } \\
\text { manufacturing context A business model } \\
\text { proposal }\end{array}$ & $\begin{array}{l}\text { Por: Mattos Nascimento, Daniel } \\
\text { Luiz; Alencastro, Viviam; Goncalves } \\
\text { Quelhas, Osvaldo Luiz; et al. (2019). }\end{array}$ & $\begin{array}{l}\text { Journal of manufacturing } \\
\text { technology management }\end{array}$ \\
\hline 04 & $\begin{array}{l}\text { Knowledge management, decision-making } \\
\text { style and organizational performance }\end{array}$ & $\begin{array}{c}\text { Abubakar, Abubakar } \\
\text { Mohammed; Elrehail, } \\
\text { Hamzah; Alatailat, Maher Ahmad; et al. } \\
\text { (2019) }\end{array}$ & $\begin{array}{l}\text { Journal of innovation \& } \\
\text { knowledge }\end{array}$ \\
\hline 05 & $\begin{array}{c}\text { Towards Industry 4.0 Mapping digital } \\
\text { technologies for supply chain management- } \\
\text { marketing integration }\end{array}$ & $\begin{array}{l}\text { Ardito, Lorenzo; Petruzzelli, Antonio } \\
\text { Messeni; Panniello, Umberto; et al. } \\
\text { (2019) }\end{array}$ & $\begin{array}{c}\text { Business process } \\
\text { management journal }\end{array}$ \\
\hline 06 & $\begin{array}{l}\text { Considering Industry } 4.0 \text { aspects in the } \\
\text { supply chain for an SME }\end{array}$ & $\begin{array}{l}\text { Bar, Kristina; Herbert-Hansen, Zaza } \\
\text { Nadja Lee; Khalid, Waqas (2018). }\end{array}$ & $\begin{array}{l}\text { Production engineering- } \\
\text { research and development }\end{array}$ \\
\hline 07 & $\begin{array}{l}\text { Complexity reduction in engineer-to-order } \\
\text { industry through real-time capable production } \\
\text { planning and control }\end{array}$ & $\begin{array}{c}\text { Rauch, Erwin; Dallasega, Patrick; Matt, } \\
\text { Dominik T.(2018) }\end{array}$ & $\begin{array}{l}\text { Production engineering- } \\
\text { research and development }\end{array}$ \\
\hline 08 & $\begin{array}{c}\text { The impact of marketing innovation on the } \\
\text { competitiveness of enterprises in the context } \\
\text { of industry } 4.0\end{array}$ & $\begin{array}{c}\text { Ungerman, Otakar; Dedkova, } \\
\text { Jaroslava; Gurinova, Katerina (2018) }\end{array}$ & Journal of competitiveness \\
\hline 09 & $\begin{array}{l}\text { What do we know about manufacturing } \\
\text { reshoring? } \\
\text {. }\end{array}$ & $\begin{array}{c}\text { Barbieri, Paolo; Ciabuschi, } \\
\text { Francesco; Fratocchi, Luciano; et al } \\
\text { (2018) }\end{array}$ & $\begin{array}{c}\text { Journal of global operations } \\
\text { and strategic sourcing }\end{array}$ \\
\hline 10 & $\begin{array}{l}\text { How does Industry } 4.0 \text { contribute to } \\
\text { operations management? }\end{array}$ & $\begin{array}{c}\text { Fettermann, Diego Castro; as } \\
\text { Cavalcante, Caroline Gobbo; de } \\
\text { Almeida, Tatiana Domingues; et al. } \\
\text { (2018) }\end{array}$ & $\begin{array}{l}\text { Journal of industrial and } \\
\text { production engineering }\end{array}$ \\
\hline 11 & $\begin{array}{l}\text { Additive manufacturing in the wood-furniture } \\
\text { sector: Sustainability of the technology, } \\
\text { benefits and limitations of adoption }\end{array}$ & $\begin{array}{l}\text { Murmura, Federica; Bravi, Laura } \\
\text { (2018) }\end{array}$ & $\begin{array}{l}\text { Journal of manufacturing } \\
\text { technology management }\end{array}$ \\
\hline 12 & $\begin{array}{l}\text { Procurement 4.0: factors influencing the } \\
\text { digitisation of procurement and supply chains }\end{array}$ & $\begin{array}{c}\text { Bienhaus, Florian; Haddud, Abubaker } \\
\text { (2018) }\end{array}$ & $\begin{array}{c}\text { Business process } \\
\text { management journal }\end{array}$ \\
\hline 13 & $\begin{array}{l}\text { Empirical assessment of the future adequacy } \\
\text { of value stream mapping in manufacturing } \\
\text { industries }\end{array}$ & $\begin{array}{c}\text { Lugert, Andreas; Batz, } \\
\text { Aglaya; Winkler, Herwig (2018) }\end{array}$ & $\begin{array}{l}\text { Journal of manufacturing } \\
\text { technology management }\end{array}$ \\
\hline 14 & $\begin{array}{l}\text { Sustainable industrial value creation: benefits } \\
\text { and challenges of industry } 4.0\end{array}$ & $\begin{array}{c}\text { Kiel, Daniel; Mueller, Julian } \\
\text { M.; Arnold, Christian; et al. (2017) }\end{array}$ & $\begin{array}{l}\text { International journal of } \\
\text { innovation management }\end{array}$ \\
\hline
\end{tabular}


Research, Society and Development, v. 10, n. 6, e52710613863, 2021

(CC BY 4.0) | ISSN 2525-3409 | DOI: http://dx.doi.org/10.33448/rsd-v10i6.13863

\begin{tabular}{|c|c|c|c|}
\hline 15 & $\begin{array}{l}\text { The Fourth Industrial Revolution (Industry } \\
\text { 4.0): A Social Innovation Perspective }\end{array}$ & $\begin{array}{c}\text { Morrar, Rabeh; Arman, Husam; Mousa, } \\
\text { Saeed (2017) }\end{array}$ & $\begin{array}{l}\text { Technology innovation } \\
\text { management review }\end{array}$ \\
\hline 16 & $\begin{array}{c}\text { The Macroeconomic Context of Investments } \\
\text { in the Field of Machine Tools in the Czech } \\
\text { Republic }\end{array}$ & Povolna, Lucie; Svarcova, Jena (2017) & Journal of competitiveness \\
\hline 17 & $\begin{array}{c}\text { Industry } 4.0 \text { learning factory didactic design } \\
\text { parameters for industrial engineering } \\
\text { education in south Africa }\end{array}$ & $\begin{array}{l}\text { Sackey, S. M.; Bester, A.; Adams, D. } \\
\text { (2017) }\end{array}$ & $\begin{array}{l}\text { South african journal of } \\
\text { industrial engineering }\end{array}$ \\
\hline 18 & $\begin{array}{l}\text { Cyber physical systems for predictive } \\
\text { production systems }\end{array}$ & $\begin{array}{l}\text { Lee, Jay; Jin, Chao; Bagheri, Behrad } \\
\text { (2017) }\end{array}$ & $\begin{array}{l}\text { Production engineering- } \\
\text { research and development }\end{array}$ \\
\hline 19 & $\begin{array}{c}\text { Methodologies of knowledge discovery from } \\
\text { data and data mining methods in mechanical } \\
\text { engineering }\end{array}$ & $\begin{array}{l}\text { Rogalewicz, Michal; Sika, Robert } \\
\text { (2016). }\end{array}$ & $\begin{array}{l}\text { Management and } \\
\text { production engineering } \\
\text { review }\end{array}$ \\
\hline 20 & $\begin{array}{l}\text { Industrial engineering curriculum in industry } \\
4.0 \text { in a south african context }\end{array}$ & Sackey, S. M.; Bester, A. (2016) & $\begin{array}{l}\text { South african journal of } \\
\text { industrial engineering }\end{array}$ \\
\hline
\end{tabular}

Fonte: Web of Science (2020).

Com o auxílio do software VOSViewer foi possível mapear os principais assuntos de interesse separados por cluster que representados graficamente por conjunto de nós. Trata-se de uma relação de co-ocorrência entre duas palavras-chave, ou seja, o número de artigos que tem palavras-chave em comum forma um nó que tem o tamanho variando de acordo com a frequência de ocorrência. Observa-se que quanto mais próximos se encontram os nós maiores serão a relação entre eles. Dentre os principais assuntos destacam-se: industry 4.0, management, future, big data, innovation, tecnology e internet conforme mapa de citação mostrado na Figura 1.

Figura 1. Mapa de assuntos relacionados ao tópico "Indústria 4.0".

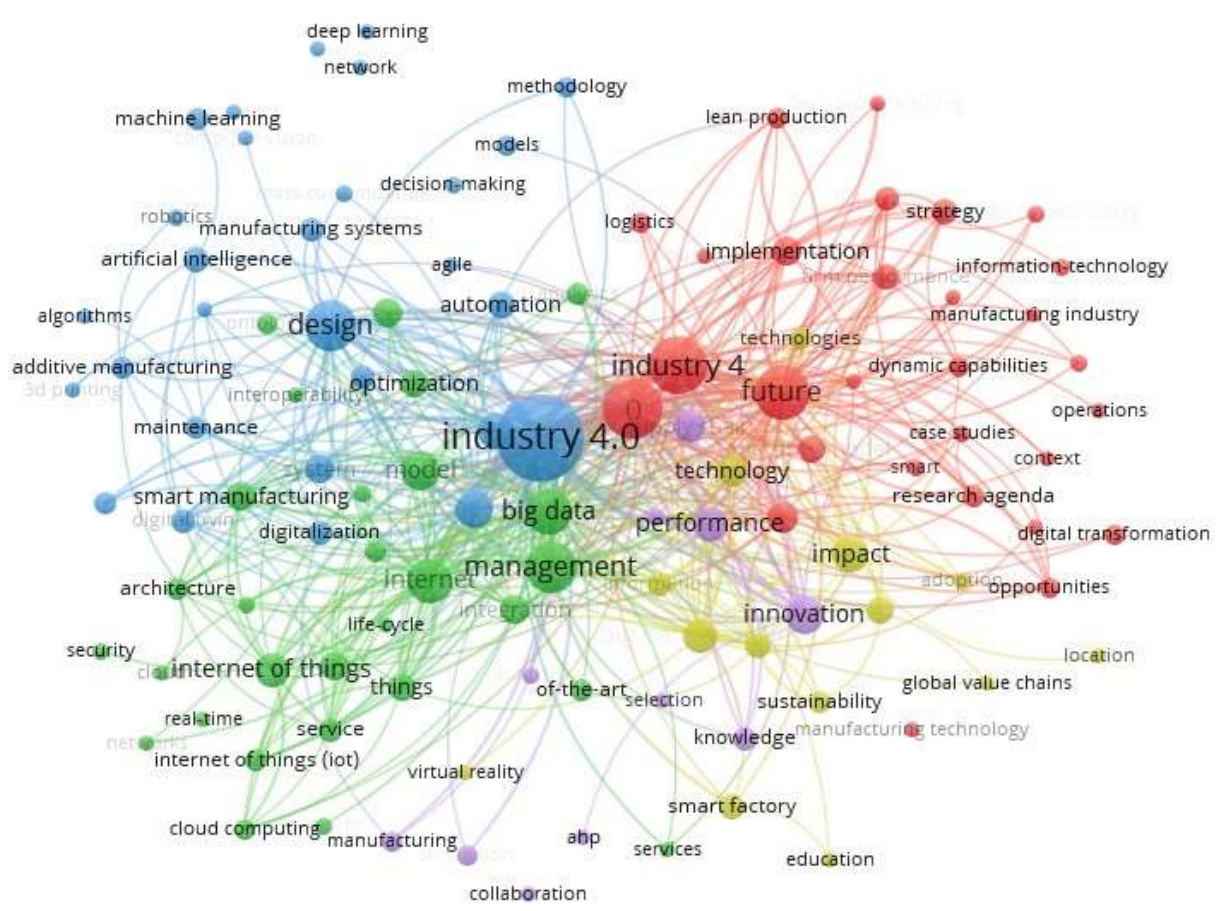

Fonte: Web of Science, Software Vos View (2020).

Os indicadores bibliométricos evidenciaram que a indústria 4.0 está relacionada à aplicação de tecnologias, ferramentas e princípios específicos. Pode ser entendida como um grande processo marcado pela automação de processos de 
digitalização e uso de ferramentas de tecnologia da informação, mas que não se limitam em suas utilizações. Dentre essas tecnologias é possível destacar: Big data \& analytcs, internet of things, Cyber Physical Systems - CPS, robôs autônomos, simulação e realidade aumentada (Lu, 2017; Wang \& Wang, 2016). A Tabela 2, evidencia as principais tecnologias utilizadas pela indústria 4.0 .

Tabela 2. Principais tecnologias da indústria 4.0.

\begin{tabular}{|c|c|c|}
\hline Tecnologias & Definição & Autor \\
\hline $\begin{array}{l}\text { Internet of things } \\
\text { (IoT) }\end{array}$ & $\begin{array}{l}\text { A IoT refere-se a interconexão entre o ambiente físico e o digital, por meio de } \\
\text { sensores e softwares que conduzem a conectividade. Permite que objetos e } \\
\text { mecanismos sejam detectados em meio à produção fornecendo uma linha de } \\
\text { produção mais eficiente e autônoma. }\end{array}$ & KANG,2016 \\
\hline $\begin{array}{l}\text { Big Data \& } \\
\text { Analytics }\end{array}$ & $\begin{array}{l}\text { É um sistema de análise de dados com características específicas em termos de } \\
\text { precisão, quantidade, velocidade, valor e diversidade, que tem como objetivo } \\
\text { capturar, transmitir, compartilhar e armazenar dados em diferentes ambientes e } \\
\text { cenários apresentados. Desempenha um papel importante na otimização da } \\
\text { qualidade e melhoria das atividades de produção na Indústria 4.0. }\end{array}$ & $\begin{array}{c}\text { WANG, 2016; } \\
\text { LIU E XUN, } 2017\end{array}$ \\
\hline $\begin{array}{l}\text { Cyber Physical } \\
\text { Systems - CPS }\end{array}$ & $\begin{array}{l}\text { O CPS é a integração de processos físicos com processos computacionais. } \\
\text { Computadores incorporados e redes de comunicação monitoram (do físico ao } \\
\text { cibernético) e controlam (do cibernético ao físico) os processos físicos, } \\
\text { geralmente em um circuito fechado onde elementos físicos e computacionais } \\
\text { interagem mutuamente. }\end{array}$ & $\begin{array}{l}\text { FRANK et al, } \\
2019\end{array}$ \\
\hline $\begin{array}{l}\text { Manufatura } \\
\text { Aditiva }\end{array}$ & $\begin{array}{l}\text { A manufatura aditiva tornou-se uma das tecnologias de desenvolvimento mais } \\
\text { dinâmico relacionadas à indústria } 4.0 \text { nos últimos anos. O uso de impressoras } \\
\text { 3D relativamente baratas e amplamente disponíveis possibilita a rápida } \\
\text { prototipagem e produção sob demanda de vários elementos e detalhes } \\
\text { exclusivos. }\end{array}$ & OKARMA,2020 \\
\hline Robôs autônomos & $\begin{array}{l}\text { A automação inteligente, incluindo robótica, é uma das tendências atuais na } \\
\text { fabricação industrial no contexto da "Indústria 4.0", onde os sistemas ciber- } \\
\text { físicos controlam a produção em fábricas automatizadas ou semi- } \\
\text { automatizadas. Os robôs são substitutos perfeitos para uma força de trabalho } \\
\text { qualificada para algumas tarefas repetíveis, gerais e estrategicamente } \\
\text { importantes. }\end{array}$ & $\begin{array}{l}\text { PÉREZ, L et al, } \\
2020\end{array}$ \\
\hline Simulação & $\begin{array}{l}\text { A simulação ajuda a avaliar os efeitos e impactos das ações no modo de } \\
\text { segurança, melhora o aprendizado e as tomadas de decisão. As mudanças de } \\
\text { comportamento nas configurações de máquinas, fluxo de processos e projetos } \\
\text { de plantas podem ser observadas no ambiente, assim como a eficácia das } \\
\text { mudanças pode ser testada sem que sejam realizadas no mundo real. }\end{array}$ & $\begin{array}{l}\text { IBRAHIM,A et al, } \\
2019\end{array}$ \\
\hline $\begin{array}{l}\text { Realidade } \\
\text { aumentada }\end{array}$ & $\begin{array}{l}\text { A realidade aumentada pode ser um elemento essencial na indústria inteligente } \\
\text { para execuções de operações, informações em tempo real são fornecidas aos } \\
\text { trabalhadores para melhorar os procedimentos de tomada de decisão. Permite } \\
\text { que os usuários simulem e explorem Interativamente. A produção é realizada } \\
\text { recriando um mapeamento realista do processo de fabricação. }\end{array}$ & VAIDYA, 2018 \\
\hline
\end{tabular}

Fonte: Kang (2016); Wang (2016); Liu e Xun (2017); Frank et al (2019); Okarma (2020); Pérez, L et al, (2020); Ibrahim, A et al (2019); Vaidya, 2018 (2020)

\subsection{Relação da indústria 4.0 e a indústria calçadista}

Após o primeiro refino na base utilizando apenas o tópico "Industry 4.0" com preferência nos artigos publicados nos últimos cinco anos, foi feito um novo refino buscando correlacionar os temas indústria 4.0 e indústria calçadista. Para tanto, usamos os tópicos “Industry 4.0" e "Footwear Industry" na base de dados Web of Science, conforme o Gráfico 2. 
Research, Society and Development, v. 10, n. 6, e52710613863, 2021

(CC BY 4.0) | ISSN 2525-3409 | DOI: http://dx.doi.org/10.33448/rsd-v10i6.13863

Gráfico 2: Número de publicações dos últimos cinco anos.

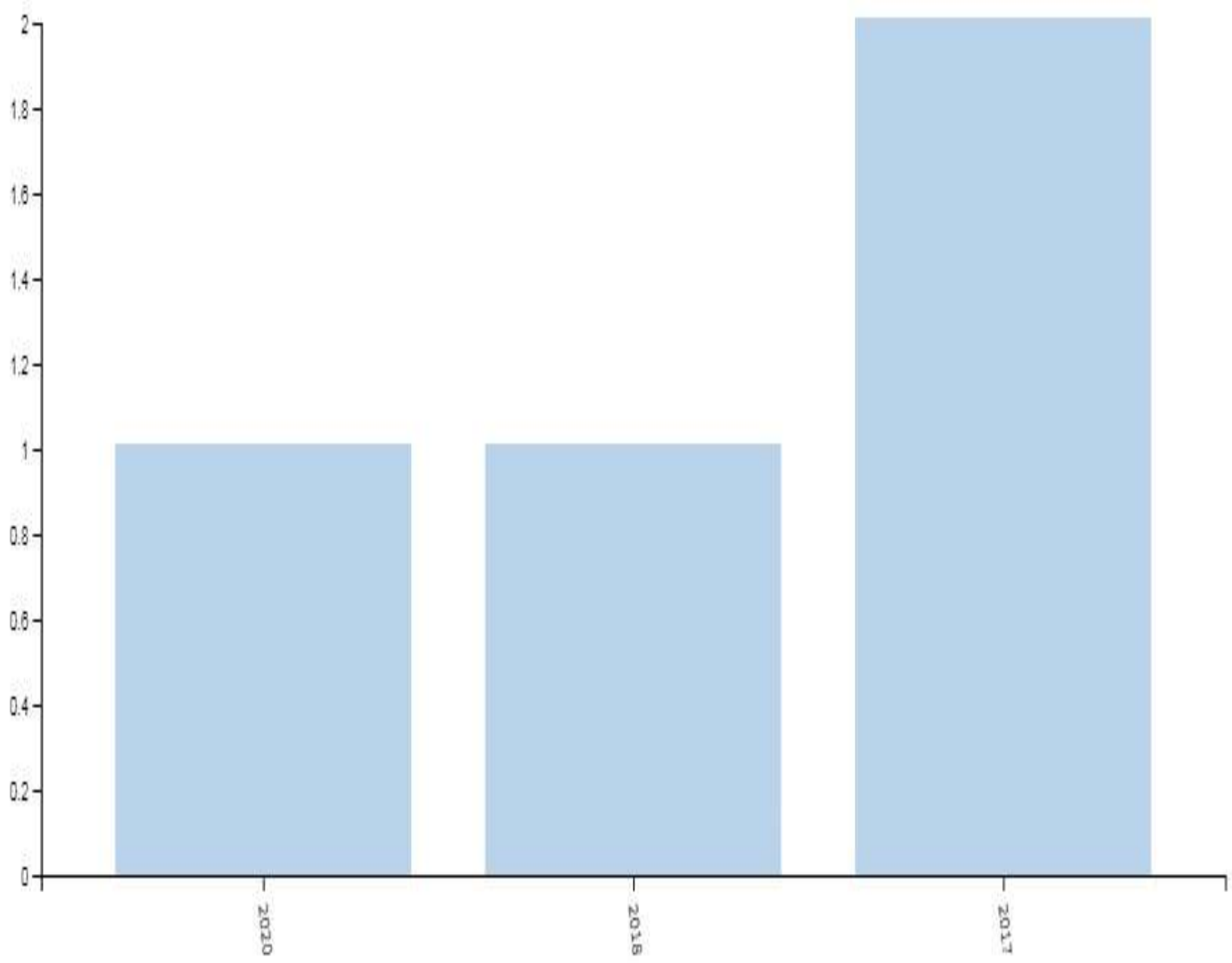

Fonte: Web of Science (2020).

A nova revisão sistemática da literatura foi realizada com o objetivo de identificar o grau de relevância da união dos dois assuntos nos últimos 5 anos. De acordo com o Gráfico 2, constata-se que as publicações científicas iniciaram apenas em 2017 com dois artigos publicados mostrando, assim, um início nas pesquisas. Novamente, a base Web of Science realizou a distribuição dos artigos por categoria e a quantidade de citações por campo do conhecimento, conforme a Tabela 3. 
Tabela 3. Distribuição de citações por campo do conhecimento.

\begin{tabular}{|c|c|c|}
\hline Campo: Categorias Da Web Of Science & Contagem De Registros & $\mathbf{\%}$ de $\mathbf{4}$ \\
\hline COMPUTER SCIENCE INTERDISCIPLINARY & 2 & $50.000 \%$ \\
\hline ENGINEERING MULTIDISCIPLINARY & 2 & $50.000 \%$ \\
\hline CHEMISTRY MULTIDISCIPLINARY & 1 & $25.000 \%$ \\
\hline $\begin{array}{c}\text { COMPUTER SCIENCE INFORMATION } \\
\text { SYSTEMS }\end{array}$ & 1 & $25.000 \%$ \\
\hline ENGENEERING MANUFACTURING & 1 & $25.000 \%$ \\
\hline MATERIAL SCIENCE MULTIDISCIPLINARY & 1 & $25.000 \%$ \\
\hline $\begin{array}{c}\text { OPERATIONS RESEARCH MANAGEMENT } \\
\text { SCIENCE }\end{array}$ & 1 & $25.000 \%$ \\
\hline PHYSICS APPLIED & 1 & $25.000 \%$ \\
\hline
\end{tabular}

Fonte: Web of Science. (2020)

Podemos observar uma predominância nas áreas de computer Science interdisciplinar e engeneering multidisciplinar, também, dando a devida relevância, às demais categorias, ao levar em conta a quantidade de artigos publicados, destacaram as categorias selecionadas para o primeiro refino usando "industry 4.0": engineering manufacturing e operations research management Science.

Finalmente, com os dados da Web of Science dos tópicos “Industry 4.0" e "Footwear Industry" e com o auxílio do software VOSViewer foi novamente possível mapear os principais assuntos de interesse separados por cluster, conforme a Figura 2.

Figura 2. Mapa de assuntos relacionados ao tópico "Industry 4.0" e "Footwear Industry".

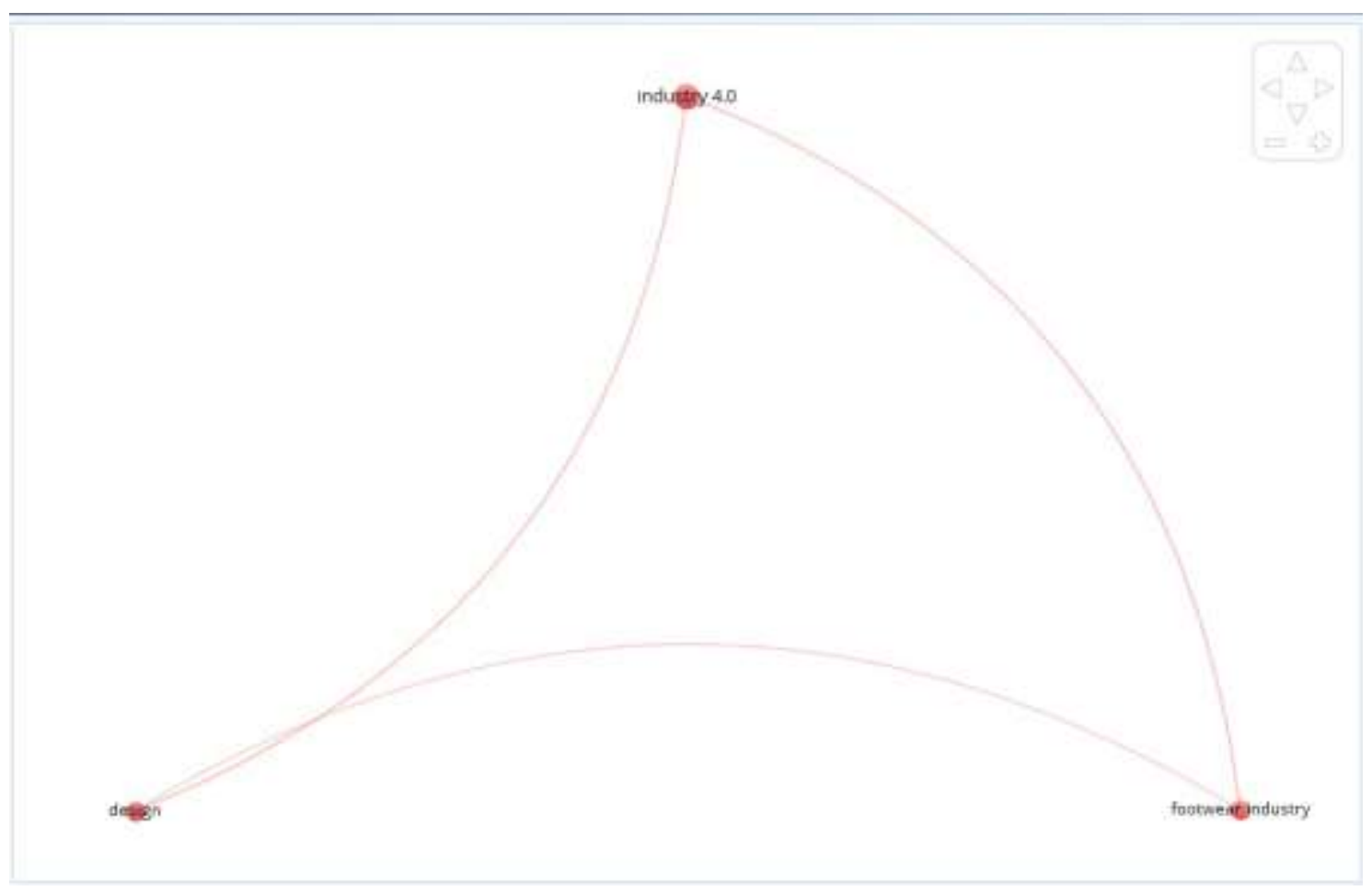

Fonte: Web of Science, VOSViewer (2020).

De acordo com a Figura 2, podemos observar a presença de apenas 1 (um) cluster formado por Industry 4.0, Footwear industry e Design. 


\section{Considerações Finais}

Este presente estudo buscou mapear, por meio de uma revisão sistemática da literatura, o desenvolvimento científico referente à quarta revolução industrial ou indústria 4.0. O assunto relativo a indústria 4.0 é recente, a pesquisa sobre o tema foi crescendo de forma mais expressiva nos últimos 5 anos, conforme revelam as publicações na base da web of Science. Ao realizar refino na base, o número inicial de artigos foi reduzido. Foi realizada a seleção de artigos mais relevantes, inicialmente pelo título, leitura de resumos e alguns casos foram realizadas a leitura integral de artigos. Sobre o aumento da produção científica sobre o tema, a pesquisa revelou interesse inicial nos estudos a partir de 2013, havendo aumento considerável no número de publicações ano pós ano, segundo os registros na base da Web of Science. Com o auxílio do software VOSViewer, foi possível mapear os principais assuntos de interesse em pesquisa, se destacando: industry 4.0, management, futute, big data, innovation, tecnology e internet.

Houve aumento considerável nas publicações em 2017 devido a disseminação e adoção dos princípios em várias indústrias, inclusive na indústria calçadista, importante no desenvolvimento regional em diversos países sendo tradicionalmente uma indústria de características relevantes como o uso abundante de mão de obra. Observa-se uma alta competitividade nessa indústria sendo que os principais assuntos correlacionados com a indústria 4.0 são: industry 4.0, footwear industry e design.

O design é uma forma dessas empresas criarem um diferencial competitivo. Outras ferramentas relacionadas ao design são importantes de serem conjuntamente estudadas, relevando uma lacuna a ser preenchida em pesquisas futuras. Além disso, percebe-se que questões relacionadas ao trabalho e ao trabalhador precisam ser exploradas no futuro, ou seja, perguntas como: Quais as implicações terão a adoção de ferramentas da indústria 4.0 no trabalho? Perguntas como essa precisa ser estudada

Diante desse contexto, percebe-se o aumento da pesquisa sobre a indústria 4.0 a cada ano. Nota-se que o interesse de compreender esse fenômeno, e de utilizar as suas ferramentas é algo essencial nas estratégias de gestão das empresas.

\section{Referências}

Frank, A., Dalenogare, L., \& Ayala, N. (2019). Industry 4.0 technologies: implmeentation patterns in manufacturing companies. International Journal of Production Economics. 210. 15. https://doi.org/ 10.1016/j.ijpe.2019.01.004

Bai, C., Dallasega, P., Orzes, G., \& Sarkis, J. (2020). Industry 4.0 technologies assessment: A sustainability perspective. International Journal of Production Economics, 229, 107776. https://doi.org/10.1016/j.ijpe.2020.107776

Bär, K., Herbert-Hansen, Z. N. L., \& Khalid, W. (2018). Considering Industry 4.0 aspects in the supply chain for an SME. Production Engineering, 12 (6), 747-758. 10.1007/s11740-018-0851-y

Chang, C. W. (2020). Evaluation of smart alarm systems for industry 4.0 technologies. Applied Sciences (Switzerland), 10 (6). 2022. https://doi.org/10.3390/app10062022

Di Roma, A. (2017). Footwear Design. The paradox of "tailored shoe" in the contemporary digital manufacturing systems. The Design Journal, 20 (sup1), S2689-S2699. http://doi.org/10.1080/14606925.2017.1352780.

Garzoni, A., De Turi, I., Secundo, G., \& Del Vecchio, P. (2020). Fostering digital transformation of SMEs: a four levels approach. Management Decision. 58. 1543. https://www.emerald.com/insight/content/doi/10.1108/MD-07-2019-0939/full/html

Gil, M., Wróbel, K., Montewka, J., \& Goerlandt, F. (2020). A bibliometric analysis and systematic review of shipboard Decision Support Systems for accident prevention. Safety Science, 128, 104717. https://doi.org/10.1016/j.ssci.2020.104717

Ibrahim, A., Kadir, T. A. A., \& Kamaludin, A. (2019). Industry 4.0: Eyeing The Future via Simulation. IOP Conference Series: Materials Science and Engineering. 769. 012001. https://iopscience.iop.org/article/10.1088/1757-899X/769/1/012001

Janson, D., Newman, S. T., \& Dhokia, V. (2019). Next generation safety footwear. Procedia Manufacturing, 38, 1668-1677. https://doi.org/10.1016/j.promfg.2020.01.117

Kang, H. S. (2016). Smart manufacturing: Past research, present findings, and future directions. International Journal of Precision Engineering and Manufacturing-Green Technology, 3 (1), 111-128. https://doi.org/10.1007/s40684-016-0015-5 
Research, Society and Development, v. 10, n. 6, e52710613863, 2021

(CC BY 4.0) | ISSN 2525-3409 | DOI: http://dx.doi.org/10.33448/rsd-v10i6.13863

Kipper, L. M., Furstenau, L. B., Hoppe, D., Frozza, R., \& Iepsen, S. (2020). Scopus Scientific mapping production in industry 4.0 (2011-2018): a bibliometric analysis. International Journal of Production Research, 58 (6), 1605-1627. https://www.tandfonline.com/doi/full/10.1080/00207543.2019.1671625

Lasi, H., Fettke, P., Kemper, H. G., Feld, T., \& Hoffmann, M. (2014). Industry 4.0. Business and Information Systems Engineering, 6 (4), 239-242. http://link.springer.com/10.1007/s12599-014-0334-4

Liu, Y., \& Xu, X. (2017). Industry 4.0 and cloud manufacturing: A comparative analysis. Journal of Manufacturing Science and Engineering, 139 (3), 034701. http://doi.org/ 10.1115/1.4034667

Lu, Y. (2017). Industry 4.0: a survey on technologies, applications and open research issues. Journal ofIndustrial Information Integration, 6, 1-10. http://doi.org/ 10.1016/j.jii.2017.04.005

Okarma, K., \& Fastowicz, J. (2020). Improved quality assessment of colour surfaces for additive manufacturing based on image entropy. Pattern Anal Applic, 23, 1035-1047. http://doi.org/ 10.1007/s10044-020-00865-w

Pérez, L., Rodríguez-Jiménez, S., Rodríguez, N., Usamentiaga, R., \& García, D. F. (2020). Digital Twin and Virtual Reality Based Methodology for MultiRobot Manufacturing Cell Commissioning. Appied Science. 10(10), 3633; https://doi.org/10.3390/app10103633

Vaidya, S., Ambad, P., \& Bhose, S. (2018). Industry 4.0: A glimpse. Procedia manufacturing. 20. 233-238. https://doi.org/10.1016/j.promfg.2018.02.034

Wang, L., \& Wang, G. (2016). Big data in cyber-physical systems, digital manufacturing and Industry 4.0. International Journal of Engineering and Manufacturing (IJEM), 6 (4), 18. http://doi.org/ 10.5815/ijem.2016.04.01 